\title{
Total Synthesis of Pikromycin and Related Macrolide Antibiotics
}

\author{
Han-Young Kang \\ Department of Chemistry, Chungbuk National University, Cheongju, Chungbuk 361-763, Republic of Korea \\ (hykang@chungbuk.ac.kr)
}

\begin{abstract}
The Pikromycin biosynthetic pathway has been attracted attention in connection with the possibility of developing combinatorial biosynthesis because it has been known to produce a variety of macrolide antibiotics. Streptomyces venezuelae, one of the most well-known organisms to produce macrolide antibiotics, produces methymycin and pikromycin families of macrolide antibiotics. The Pik PKS (polyketide synthase), a gigantic enzyme responsible for producing all the compounds belongs to these families, generates both of methymycin and pikromycin families of macrolides which contain 12- and 14-membered macrolactones, respectively. During the pikromycin biosynthetic pathway 10-deoxymethynolide (a 12-membered macrolactone) and narbonolide (a 14-membered macrolactones) are produced first. They are converted to methymycin, pikromycin, and other macrolide antibiotics by the action of Des and PikC (a P450 hydroxylase). Total of eight macrolide antibiotics are formed from the pikromycin pathway.

We have investigated the synthesis of the macrolide antibiotics from the pikromycin pathway not only because securing these macrolides in reasonable quantities might be critically helpful to study the biosynthetic pathway but also because they have unique structural features to attract synthetic chemists' attention. Our successful synthetic efforts for developing general routes to the macrolide antibiotics including methymycin and pikromycin will be presented.
\end{abstract}

\section{References}

1. Xuan, R; Oh, H.-S.; Lee, Y.; Kang, H.-Y. J. Org. Chem. 2008, 73, 1456-1461.

2. Park, J. W.; Oh, H.-S.; Jung, W. S.; Park, S. R.; Han, A. R.; Ban, Y.-H.; Kim, E. J.; Kang, H.-Y.; Yoon, Y. J. Chem. Commun. 2008, 5782-5784.

3. Oh, H.-S.; Xuan, R.; Kang, H.-Y. Org. Biomol. Chem. 2009, 7, 4458-4463.

4. Oh, H.-S.; Kang, H.-Y. Tetrahedron 2010, 66, 4307-4317.

5. Oh, H.-S.; Kang, H.-Y. J. Org. Chem. 2012, 77, 1125-1130. 